\title{
mTOR inhibitors response and mTOR pathway in pancreatic neuroendocrine tumors
}

\author{
Simona Falletta1, Stefano Partelli2, Corrado Rubini³, Dominik Nann4, Andrea Doria³, \\ Ilaria Marinoni4, Vanessa Polenta², Carmelina Di Pasquale1, Ettore degli Uberti1, \\ Aurel Perren4, Massimo Falconi² and Maria Chiara Zatelli1 \\ 1Department of Medical Science, Section of Endocrinology and Internal Medicine, University of Ferrara, \\ Ferrara, Italy \\ 2Pancreatic Surgery Unit, Pancreas Translational and Research Institute, San Raffaele Hospital, \\ Vita-Salute San Raffaele University, Milan, Italy \\ ${ }^{3}$ Department of Biomedical Sciences and Public Health, Polytechnic University of Marche, Ancona, Italy \\ ${ }^{4}$ Institut fur Pathologie, University of Bern, Bern, Switzerland
}

Correspondence should be addressed to M C Zatelli Email

ztImch@unife.it

\begin{abstract}
Medical therapy of pancreatic neuroendocrine tumors (P-NET) may take advantage of Everolimus treatment. However, the extent of therapeutic response cannot be predicted. This study was aimed to identify the possible predictive markers of response to Everolimus in P-NET. We found that Everolimus reduced the cell viability and induced apoptosis in primary cultures of 6 P-NET (P-NET-R), where the proliferative and antiapoptotic effects of IGF1 were blocked by Everolimus. On the contrary, 14 P-NET primary cultures (P-NET-NR) were resistant to Everolimus and IGF1, suggesting an involvement of PI3K/AKT/mTOR pathway in the mechanism of resistance. The response to Everolimus in vitro was associated with an active AKT/mTOR pathway and seemed to be associated with a greater clinical aggressiveness. In addition, a patient sensitive to Everolimus in vitro was sensitive to this drug in vivo also and showed a positive $\mathrm{p}$-AKT immunohistochemistry (IHC) at tissue level. Similarly, a patient resistant to Everolimus treatment after surgery was not sensitive to the drug in vitro and had a negative $\mathrm{p}$-AKT IHC staining. Therefore, present data confirm that P-NET primary cultures may be considered a model for testing medical treatment efficacy and that IHC characterization of $\mathrm{p}$-AKT might help in identifying human P-NET who can benefit from Everolimus treatment. These data encourage conducting a prospective multicenter study involving different groups of P-NET patients treated with Everolimus.
\end{abstract}

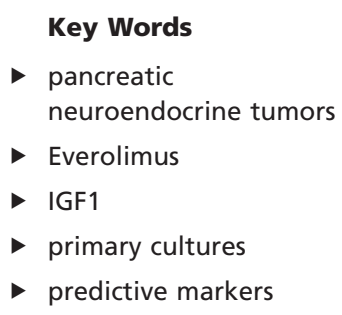

Endocrine-Related Cancer (2016) 23, 883-891

\section{Introduction}

Pancreatic neuroendocrine tumors (P-NETs) are heterogeneous neoplasms originating from pancreatic neuroendocrine cells (Singh et al. 2015, Berardi et al. 2016), representing $1-4 \%$ of all pancreatic malignancies, with an increasing estimated annual incidence of 1 case/100,000 inhabitants (Al-Kurd et al. 2014). Based on the hormonal secretion, P-NETs are divided into functioning and nonfunctioning tumors, depending 
on the clinical manifestation of a syndrome related to specific hormonal hypersecretion (Halfdanarson et al. 2008, Missiaglia et al. 2010, Ro et al. 2013). Radical surgery represents the first-line treatment for localized disease, whereas it is often not feasible for locally advanced or metastatic tumors (Vallea et al. 2014). The most effective pharmaceutical option in functioning tumors is represented by somatostatin analogues (SSAs) that are useful to achieve symptomatic control. These drugs have also been recommended for the control of P-NET growth by recent guidelines (Pavel et al. 2016).

The mammalian target of rapamycin (mTOR) is a serine threonine kinase located downstream the PI3K/AKT signaling pathway that plays a crucial role in mediating basic cellular functions (Jiang \& Liu 2008). The PI3K/AKT/mTOR pathway is constitutively activated in different NETs, including those of pancreatic origin (Capdevila et al. 2011). The expression of MTOR pathway components has been suggested to correlate with clinical behavior and responsiveness to mTOR inhibitors (Righi et al. 2010, Qian et al. 2013, Zatelli et al. 2016). The mTOR inhibitor has recently shown antitumor activity in advanced, well-differentiated and moderately differentiated P-NETs (Yao et al. 2011), but resistance to this treatment may develop. However, the mechanisms regulating the sensitivity/resistance to mTOR inhibitors are still scantly elucidated, and predictive biomarkers of Everolimus efficacy in P-NET are still lacking. We recently demonstrated that responsiveness to Everolimus in human bronchial carcinoid primary cultures correlates with an active AKT/mTOR signaling pathway (Gagliano et al. 2013). However, PI3K/AKT/mTOR signaling does not always predict the sensitivity to mTOR inhibitors and is difficult to assess (Zatelli et al. 2016). Therefore, the aim of this study was to investigate the possible predictors of sensitivity to Everolimus in P-NET, to identify patients who may benefit from treatment avoiding useless side effects in resistant patients.

\section{Materials and methods}

\section{Human pancreatic neuroendocrine tumors}

Twenty samples, including 18 primary P-NETs and 2 lymph node metastases, were derived from 16 patients (10 males and 6 females; mean age $58.6 \pm 4.2$ years) diagnosed with P-NET, who were operated on at the University of Ancona (Pancreatic Surgery Unit, Department of Surgery) and at the University of Ferrara (Section of Endocrinology and Clinical Surgery). Patients and tumor characteristics are displayed in Table 1. Among the investigated patients, 13 did not receive medical therapy before surgery; 3 had been previously treated with somatostatin analogues (SSA) alone (1 patient), in association with chemotherapy (1 patient) or with Everolimus (1 patient, who showed disease progression after being treated with capecitabine).

Most P-NETs were diagnosed as G1 or G2 (14/16), displaying a Ki67 $\leq 10 \%$, with pauci cellular focal necrosis

Table 1 Clinical characteristics of P-NET patients.

\begin{tabular}{|c|c|c|c|c|c|}
\hline Patient no. & Sex & Age & Hormonal secretion & Diagnosis & TNM \\
\hline 1 & $\mathrm{~F}$ & 67 & NF & NET G2 & T3 N1 M1 \\
\hline 2 & $\mathrm{M}$ & 74 & NF & NEC G3a & T2 NO M1 \\
\hline 3 & $\mathrm{M}$ & 53 & NF & NET G2 & T2 N1 M0 \\
\hline 4 & $\mathrm{M}$ & 76 & NF & NET G1 & T1 No Mo \\
\hline $5^{b}$ & $\mathrm{~F}$ & 38 & NF & NET G2 & T4 NO MO \\
\hline $6^{c}$ & $\mathrm{M}$ & 56 & NF & NEC G3 & T3 N1 M1 \\
\hline $7 b, c$ & $\mathrm{~F}$ & 65 & NF & NET G2 & T3 N1 M1 \\
\hline 8 & $\mathrm{~F}$ & 32 & NF & NET G1 & T2 NO MO \\
\hline 9 & $\mathrm{M}$ & 77 & NF & NET G1 & T1 No M0 \\
\hline 10 & $\mathrm{~F}$ & 74 & NF & NET G2 & T2 NO MX \\
\hline 11 & $\mathrm{M}$ & 23 & Insulinoma & NET G2 & T3 N1 M0 \\
\hline $12^{d}$ & $\mathrm{M}$ & 65 & NF & NET G1 & T1 No MO \\
\hline 13 & $\mathrm{M}$ & 75 & Insulinoma & NET G1 & T1 NO MO \\
\hline 14 & $\mathrm{~F}$ & 65 & NF & NET G1 & T1 NO MO \\
\hline $15^{d}$ & $\mathrm{M}$ & 45 & Insulinoma & NET G1 & T1 No MO \\
\hline 16 & $\mathrm{M}$ & 53 & NF & NET G2 & T2 NO MO \\
\hline
\end{tabular}

\begin{tabular}{lll} 
Stage & & Diameter $(\mathrm{cm})$ \\
\cline { 1 - 1 } IV & & 4 \\
IV & & 2.8 \\
IIB & & 3.4 \\
I & & 1.5 \\
III & & 9.5 \\
IIB & & 8 \\
IV & & 2.5 \\
IB & & 3 \\
I & & 1.1 \\
IB & & 1.8 \\
IIB & & 2.5 \\
I & 1.3 \\
I & 1.9 \\
I & 2 \\
I & 0.5 \\
I & 2.5
\end{tabular}

\begin{tabular}{c} 
Grade \\
\hline 2 \\
3 \\
2 \\
1 \\
2 \\
3 \\
2 \\
1 \\
1 \\
2 \\
2 \\
1 \\
1 \\
1 \\
1 \\
2
\end{tabular}

\begin{tabular}{ccrrr}
\hline Mitosis no. & & Ki67 (\%) \\
\cline { 1 - 1 } $2 / 50$ & & 8 \\
$42 / 10$ & & 65 \\
$2 / 10$ & & 9 \\
$2 / 10$ & & 2 \\
$2 / 10$ & & 10 \\
$5 / 10$ & & 25 \\
$13 / 10$ & & 20 \\
$1 / 50$ & & 1 \\
$2 / 10$ & & 2 \\
$2 / 10$ & & 5 \\
$2 / 10$ & & 7 \\
$2 / 10$ & & 1 \\
$1 / 10$ & & 2 \\
$2 / 10$ & & 2 \\
0 & & $<2$ \\
NA & 3
\end{tabular}

NA, not available; NEC, neuroendocrine carcinoma; NET, neuroendocrine tumor; NF, non-functioning. aPoorly differentiated neuroendocrine carcinoma; bNET patients for whom 1 sample from the primary tissue and 1 sample from the lymph node metastasis were available, each generating a primary culture; cNET patients treated with Everolimus in vivo; dNET patients for whom 2 primary tissue samples were available, each generating a primary culture. 
in some cases, without lymph node $(11 / 16)$ or distant metastases (13/16).

\section{Tissue collection and primary cultures}

Tissue samples were collected following the guidelines of the local committee on human research. Informed consent of the patients was obtained for disclosing clinical investigation and performing the in vitro study. Tissue samples were stored in RNA-later solution (Sigma) for expression studies; upon arrival in the lab, they were immediately frozen in liquid nitrogen under ribonuclease (RNase)-free conditions and stored at $-80^{\circ} \mathrm{C}$ until protein isolation was performed. A portion of the fresh tissue was collected in culture medium for primary culture studies; upon arrival in the lab, the tissue was immediately processed for primary culture experiments as described previously, with minor modifications (Molè et al. 2011). Tumor cells were resuspended in F-12 with 10\% fetal bovine serum (FBS) and antibiotics (Euroclone Ltd, Wettherby, UK), seeded at $2 \times 10^{4}$ cells/well in 96-well black plates and incubated at $37^{\circ} \mathrm{C}$ in a humidified atmosphere of $5 \% \mathrm{CO}_{2}-95 \%$ air, as described previously (Zatelli et al. $2010 a$ ). After approximately $18 \mathrm{~h}$, cells were treated with test compounds, with further evaluation of cell viability and/or caspase activity.

\section{Compounds}

Everolimus was provided by Novartis; IGF1 was purchased from PeproTek (Rocky Hill, NJ, USA). All other reagents were purchased from Sigma, if not otherwise indicated.

\section{Viable cell number and caspase activation assessment}

Cell viability and caspase activation were measured as described previously (Zatelli et al. 2006, Zatelli et al. $2010 a, b)$ using the ATPlite kit (PerkinElmer Life Sciences) and the caspase-Glo 3/7 assay (Promega), respectively. Briefly, 20 P-NETs primary cultures, derived from 16 patients, were treated with or without $100 \mathrm{nM}$ Everolimus for $48 \mathrm{~h}$ in the absence or in the presence of $100 \mathrm{nM}$ IGF1. Luminescent output (relative luminescence units, RLU) was recorded after $48 \mathrm{~h}$ for each assessment by the Envision Multilable Reader (PerkinElmer). Results are expressed as mean value \pm S.E.M. percent RLU vs untreated control cells.

According to the response to Everolimus, samples were divided into primary cultures displaying a significant reduction $(P<0.05$ vs untreated cells $)$ in cell viability under Everolimus treatment, indicated as responders (P-NET-R), and into primary cultures in which Everolimus did not reduce cell viability, indicated as nonresponders (P-NET-NR).

\section{Kinase activity assay}

Protein isolation from frozen human P-NET tissues was performed as described previously (Gagliano et al. 2013). RIPA buffer (Pierce) was used to dissolve tissues that were kept in ice for $30 \mathrm{~min}$, and then centrifuged for $10 \mathrm{~min}$. Protein concentration in the supernatant was measured by the BCA Protein Assay Reagent Kit (Pierce), as described previously (Tagliati et al. 2006). Each sample was evaluated in 3-6 replicates by assessing the same total protein amount. Phosphorylated levels of IGF1R (Tyr1135/1136), AKT (Ser473), mTOR (Ser2448) and 4EBP1 (Thr37/46) were measured using the AlphaScreen SureFire assays (PerkinElmer Life Sciences). Normalization against glyceraldehyde-3-phosphate dehydrogenase (GAPDH) using the appropriate AlphaScreen SureFire assay kit was performed. Results are expressed as mean value \pm S.E.M. AlphaScreen signal (counts).

\section{Tissue microarray construction}

Paraffin blocks were available for 15 P-NET patients. Tissue microarrays (TMA) were manually assembled from formalin-fixed, paraffin-embedded tissues. Three representative $1 \mathrm{~mm}$ diameter normal and tumoral tissue cores were taken from each block, for a total of 45 specimens. Multiple $3-\mu \mathrm{m}$ sections were cut with a Leica microtome (Leica Microsystems), dried at $60^{\circ} \mathrm{C}$ and then transferred to adhesive-coated slides for immunohistochemical staining.

\section{Immunohistochemistry}

Immunohistochemistry (IHC) was performed by an anti-p-AKT (Ser473) antibody obtained by Abcam. Slides were de-paraffinized and re-hydrated in dewax solution (Leica Biosystems), and endogenous peroxidase activity was blocked with $\mathrm{H}_{2} \mathrm{O}_{2}$ solution for $4 \mathrm{~min}$. After antigen retrieval using citrate buffer, $\mathrm{pH} 6.0\left(15 \mathrm{~min}\right.$ at $\left.100^{\circ} \mathrm{C}\right)$, slides were incubated with the anti-p-AKT antibody (1:300) for $30 \mathrm{~min}$, at room temperature. Immunoreactions were revealed by using the Bond Polymer Refine Kit on the automated system BOND RX (both from Leica Biosystems). Antigen-antibody complexes were detected using the cobalt-3,3'-diaminobenzidine (Co-DAB)

Published by Bioscientifica Ltd 
as the chromogen (Leica Biosystems). Samples were counterstained with hematoxylin and mounted in Aquatex (Merck). The specificity of all reactions was validated in parallel control sections omitting the primary antibodies for each IHC run. Endometrial cancers that are known to have, in the majority of cases, a PTEN mutation leading to PI3K/AKT pathway constitutive activation were used as positive controls.

\section{Evaluation of staining of tissue slides}

Tumor staining characteristics were reviewed and interpreted independently by two pathologists (A P and D N). IHC data were visually scored. Intensity and location (nuclear and membranous) were taken into consideration. The immunostaining intensity was evaluated subjectively as being negative (0), weak (1), moderate (2) and strong (3) and then the staining was classified into two categories: negative (no immunostaining) and positive (weak-moderate and strong immunostaining), in more than $10 \%$ of tumor cells.

\section{Statistical analysis}

Results are expressed as the mean \pm s.E.M. Concerning the results of cell viability and caspase 3/7 activation experiments, a preliminary analysis was carried out to determine whether the datasets conformed to a normal distribution. The results were compared within each group and between the groups using ANOVA. If the $F$ values

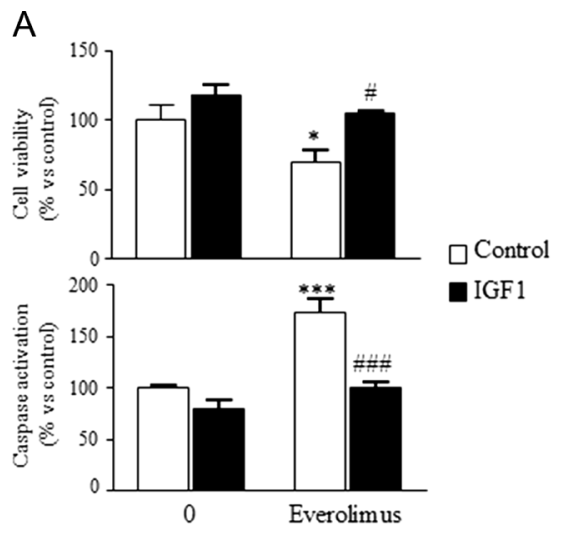

were significant $(P<0.05)$, Student's paired or unpaired $t$-test was used to evaluate individual differences between the means. $P$ values $<0.05$ were considered significant. For the AlphaScreen assays, after proof of homogeneity of variance and normality test, Student's unpaired $t$-test was used. In case of non-normal distribution, Mann-Whitney test was used.

To compare the clinicopathological features, a preliminary analysis was carried out to determine whether the datasets conformed to a normal distribution and then Mann-Whitney test was used, due to not normal distribution for Ki67, tumor size and number of mitoses. For sex, primary vs. metastatic tissue, pre-treatment with SSA, Octreoscan/68GA-PET uptake, site, TNM, stage, necrosis and tumor grade, chi-square test corrected for linearity was applied. Differences in age were assessed by the Student's $t$-test. Data were analyzed using GraphPad (Prism v-5.0).

\section{Results}

\section{Effects of Everolimus and IGF1 on P-NET primary cultures}

Six P-NET primary cultures were defined as P-NET-R and 14 P-NETs were defined as P-NET-NR. As shown in Fig. 1A (upper panel), in P-NET-R, Everolimus significantly reduced the cell viability $(-29.9 \% ; P<0.05$ vs untreated cells), an effect completely counteracted by co-incubation with IGF1. In P-NET-NR (Fig. 1B, upper panel), cell viability was significantly induced by Everolimus

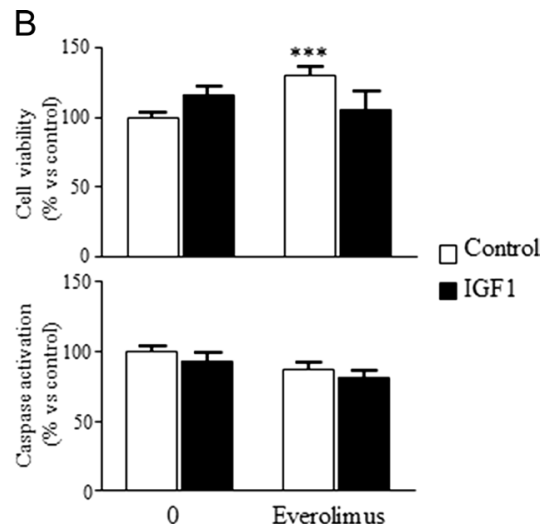

Figure 1

Effects of Everolimus and IGF1 on P-NET primary cultures. P-NET primary cultures were incubated in 96-well plates for 48 $\mathrm{h}$ in a culture medium supplemented with $100 \mathrm{nM}$ Everolimus with (black bars) or without $100 \mathrm{nM}$ IGF1 (white bars); control cells were treated with a vehicle solution. Cell viability and caspase activation of each primary culture were measured as a luminescent signal. According to the response to Everolimus in terms of cell viability inhibition, the samples were divided into 'responder' (6 samples) (A) and 'non-responder' (14 samples) (B). Data from P-NET primary cultures were evaluated independently with six replicates each, and expressed as the mean value \pm s.E.M. percent cell viability inhibition vs untreated control cells (upper panels) or as the mean value \pm S.E.M. percent caspase activation vs untreated control cells (lower panels). $* P<0.05$ and $* \star * P<0.001$ vs untreated control cells. ${ }^{P} P<0.05$ and ${ }^{\# \#} P<0.001$ vs cells treated with Everolimus alone. 
$(+30.2 \% ; P<0.001$ vs control cells) and was not affected by IGF1, alone or in combination with Everolimus.

To verify whether Everolimus modifies cell viability by influencing the apoptotic process, caspase $3 / 7$ activation was evaluated. As shown in Fig. 1A (lower panel), in P-NET-R Everolimus significantly induced caspase activation ( $+73 \% ; P<0.001$ vs untreated cells), an effect completely counteracted by co-incubation with IGF1. In P-NET-NR (Fig. 1B, lower panel), both Everolimus and IGF1 did not significantly affect caspase activation. These results indicate that P-NET may respond differently to Everolimus in vitro and that P-NET-NRs are not sensitive to IGF1 either, suggesting that IGF1 transduction pathway is not active in these tissues. Our data suggest that IGF1 pathway may be involved in the mechanism of resistance to Everolimus in vitro.

\section{IGF1/mTOR signaling pathway expression in P-NET tissues}

As IGF1 was capable of reversing the inhibitory effects of Everolimus on cell viability in P-NET-R, we investigated the levels of IGF1 downstream signaling proteins involved in mTOR pathway. Proteins isolated from frozen P-NET tissues, classified as P-NET-R and P-NET-NR on the basis of the results of primary cultures, were used to evaluate the expression levels of phosphorylated IGF1 R (p-IGF1 R), AKT (p-AKT), mTOR (p-mTOR) and 4EBP1 (p-4EBP1) by using AlphaScreen SureFire assay. As shown in Fig. 2A, we found that $\mathrm{p}-\mathrm{IGF} 1 \mathrm{R}$ protein levels were significantly $(P<0.001)>2$-fold lower in P-NET-NR compared with those in P-NET-R. Similarly, p-AKT levels were $>2$-fold lower in P-NET-NR compared with those in P-NET-R (Fig. 2B); however, statistical significance was not reached, possibly due to the small number of samples. Moreover, p-mTOR and p-4EBP1 protein levels were significantly $(P<0.02$ and $P<0.001)>2$-fold lower in P-NET-NR compared with P-NET-R (Fig. 2C and D). These data indicate that response to Everolimus in vitro is associated with an active AKT/mTOR pathway.

\section{Clinical and pathological correlations}

When the clinical characteristics of the patients were evaluated according to Everolimus responsiveness in vitro, there was no significant difference between patients with P-NET-R compared with those with P-NET-NR in terms of sex, age, primary vs metastatic tissue, pre-treatment with SSA, Octreoscan/ ${ }^{68}$ GA-PET uptake, site, TNM, stage, size, number of mitoses and necrosis. However, ki67 was significantly $(P<0.05)$ higher in P-NET-R (median 10\%) than that in P-NET-NR (median 2\%) (Fig. 3A). In addition, a trend for a linear correlation between tumor grade and response to Everolimus was found. All G3 P-NET responded to the treatment in vitro, whereas only $14 \%$ of G1 P-NET were P-NET-R (Fig. 3B).

\section{Expression of mTOR pathway components in P-NET and association with clinical outcomes}

To validate the identified putative markers of response to Everolimus in P-NET, the expression of $\mathrm{p}$-AKT protein was
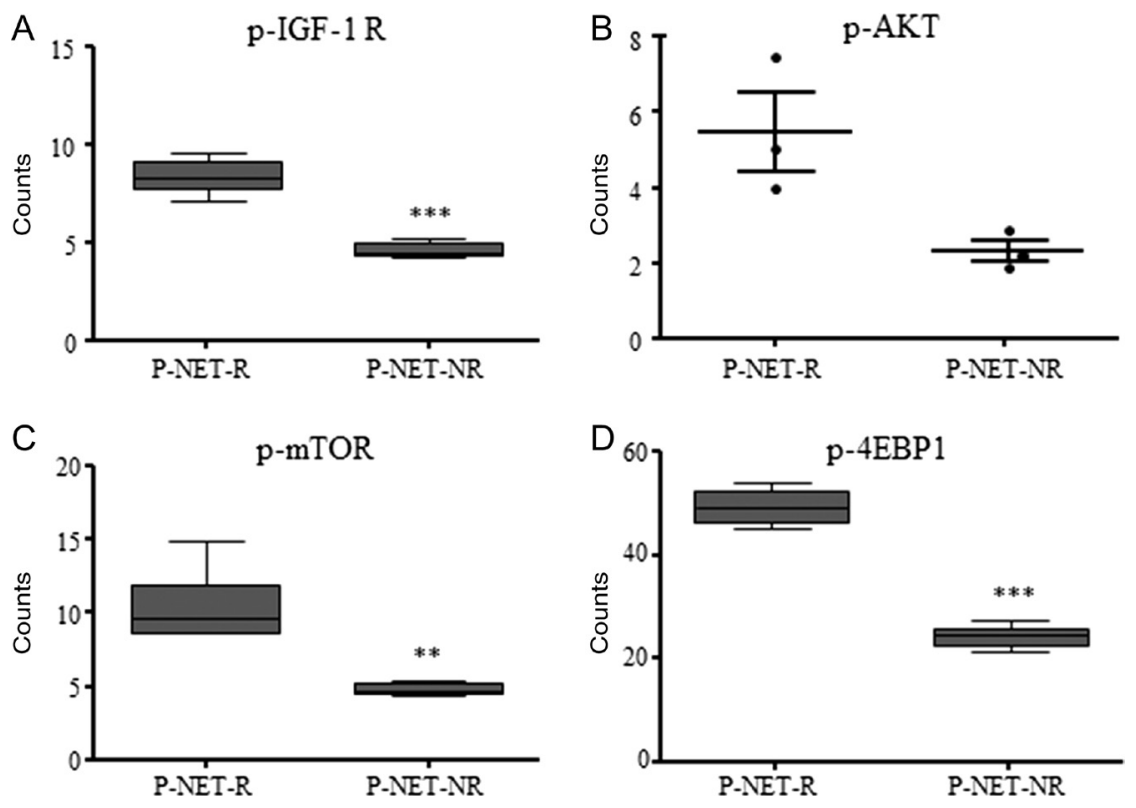

Figure 2

IGF1/mTOR signaling pathway expression in P-NET tissues. Total proteins were isolated from 20 P-NET tissues and AlphaScreen analysis for p-IGF1 R (A), p-AKT (B), p-mTOR (C) and p-4EBP1 (D) expression in the pooled P-NET R and in the pooled P-NET NR tissues was performed, as described in the 'Materials and methods' section. Values are expressed after normalization against glyceraldehyde-3-phosphate dehydrogenase (GAPDH) as AlphaScreen signal (counts). ${ }^{*} P<0.02$ and $* * * P<0.001$ vs P-NET R. 
A

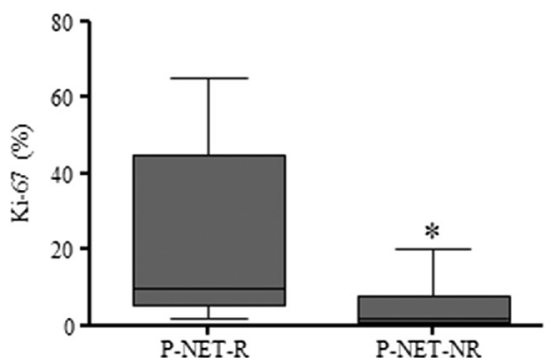

B

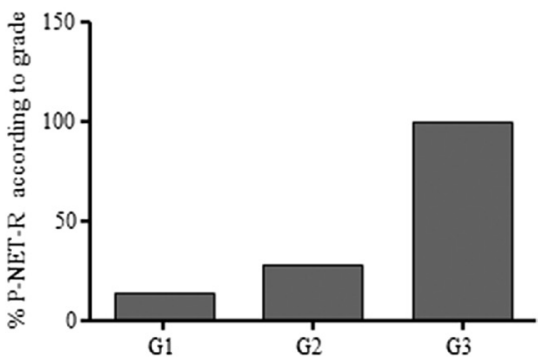

Figure 3

Patients clinical characteristics and Everolimus responsiveness in vitro. (A) Median values and range of Ki67 labelling index in P-NET-R and in P-NET-NR. ${ }^{*} P<0.05 \%$ vs P-NET-R.

(B) \% P-NET-R tissues according to tumor grade. G1 P-NET-R=1/7; G2 P-NET-R = 2/7; G3 P-NET-R = 2/2. evaluated using IHC. Four tissue samples did not provide reliable results due to technical problems. Therefore, we obtained reliable results for 11 patients, 3 P-NET-R and 8 P-NET-NR. IHC for p-AKT was positive in all the paraffin-embedded tissues that correspond in vitro to 3 P-NET-R. IHC for p-AKT was positive in 5 out of 8 paraffin-embedded tissues that corresponded in vitro to P-NET-NR. There was no statistically significant correlation between p-AKT positivity by IHC and responsiveness to Everolimus in vitro, possibly due to the low number of available samples. The response to Everolimus in vitro, as well as p-AKT protein levels in frozen tissues were also compared with the response to Everolimus in vivo, in terms of tumor stabilization/progression and survival. Two patients had been treated with Everolimus after surgery due to the presence of non-resectable liver metastases. The first patient was a 56-year-old male operated on for a well-differentiated non-functioning G3 P-NET (T3N1M1, Ki67=25\%), which turned out to be a P-NET-R in vitro. The patient displayed stabilization of the

A
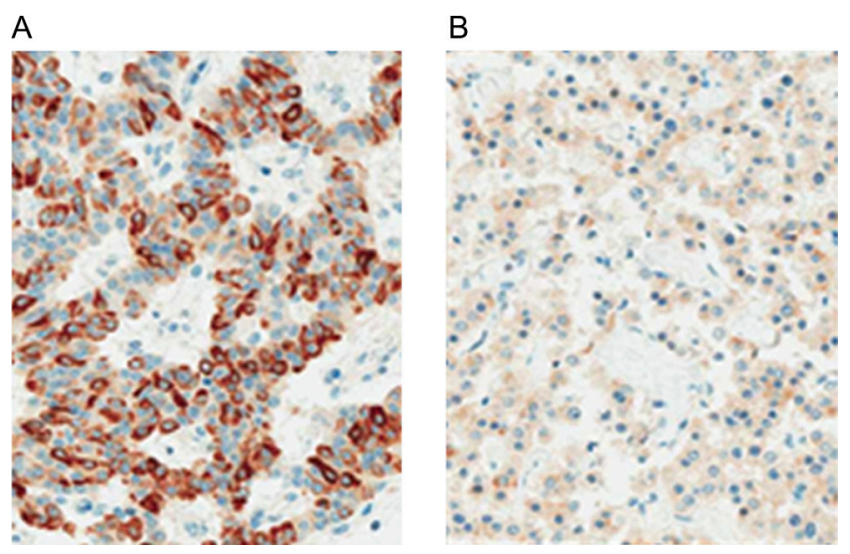

Figure 4

Association between p-AKT expression and clinical outcomes. (A) Immunohistochemical expression of $p$-AKT in a non-functioning G3 P-NET (T3N1M1), sensitive to Everolimus after surgery, defined as P-NET-R in vitro. (B) Immunohistochemical expression of $\mathrm{p}-\mathrm{AKT}$ in a non-functioning G2 P-NET (T3N1M1), resistant to Everolimus after surgery and defined as P-NET-NR in vitro. disease during Everolimus treatment, with a progressionfree survival of 21 months (still alive at the end of the study). Furthermore, he showed positive p-AKT IHC at tissue level (Fig. 4A). The second patient was a 65 -yearold female operated on for a non-functioning G2 P-NET (T3N1M1, Ki67 $=20 \%$ ), that turned out to be a P-NET-NR in vitro. The patient displayed disease progression after 12 months of treatment with Everolimus, with a survival of 21 months (still alive at the end of the study). Data from IHC showed negative p-AKT staining (Fig. 4B).

\section{Discussion}

Our study shows that P-NET primary cultures are a useful model for testing medical therapy in vitro. We also identified putative markers that may predict the response to Everolimus treatment in vivo. In our settings, 30\% of human P-NET primary cultures responded to Everolimus treatment with a significant reduction in cell viability and induction of apoptosis, whereas the remaining $70 \%$ did not. These data may seem in contrast with the demonstrated efficacy of Everolimus in prolonging PFS in advanced P-NET (Yao et al. 2011, Wolin 2014). It should be underlined that we defined as responders those primary cultures displaying a significant cell viability reduction in vitro after $48 \mathrm{~h}$. This parameter may not completely reflect the results obtained on clinical grounds in terms of PFS, which is influenced by many clinical variables and not by tumor growth only. On the other hand, our results may help in identifying those patients who may better benefit from treatment not only in terms of longer PFS but also in terms of tumor bulk reduction. Moreover, our series shows intra-patient and intra-tumor heterogeneity in primary cultures, further underlining P-NET heterogeneity. In addition, we show that the effect of Everolimus was influenced by IGF1 only in human P-NET tissues classified as responders to the drug in vitro, but not in human P-NET tissues resistant to the drug. The importance of IGF1 in regulating NET proliferation is underlined by the evidence of an enhanced and constitutive expression of IGF1 and

Published by Bioscientifica Ltd. 
IGF1R in gastroenteropancreatic NET, which leads to autocrine and paracrine growth stimulation (von Wichert et al. 2000, Höpfner et al. 2008). Moreover, we previously demonstrated that IGF1 exerts protective effects toward the anti-proliferative action of Everolimus in a group of medullary thyroid carcinoma primary cultures (Gentilin et al. 2015). Therefore, these data suggest that IGF1 pathway may play an important role in the development of Everolimus resistance. The latter represents a relevant issue because previous evidence demonstrated that some patients do not benefit from Everolimus treatment, due to the development of primary or secondary acquired resistance to this drug (Fazio 2015), despite a significant prolongation of PFS shown by the RADIANT-3 trial in a significant number of patients with P-NET (Yao et al. 2011). However, the precise mechanism underlying Everolimus resistance remains unknown (Zatelli et al. 2016).

Everolimus inhibits mTOR activity by interacting with the mTORC1 complex but not with mTORC2 complex. Selective blockade of mTORC1 by Everolimus leads to an increase in mTORC2 activity and results in a positive feedback activation of AKT, by phosphorylation on Ser473, and of IGF1/IGFR signaling due to inhibition of the S6K negative feedback (O'Reilly et al. 2006). As an evident association between the expression levels of PI3K/AKT/ mTOR components and response to Everolimus in vitro was observed in bronchial carcinoids and in nonfunctioning pituitary adenomas (Zatelli et al. 2010a, Gagliano et al. 2013), we hypothesized that the investigation of mTOR pathway components could be useful to predict the responsiveness to Everolimus treatment in P-NET. In this setting, AlphaScreen data show that P-NET-R tissues display higher levels of p-IGF1R, p-AKT, p-mTOR and p-4EBP1 compared with those of P-NET-NR tissues, supporting the hypothesis that the expression of these proteins could be used to separate 'responder' tissues from 'resistant' tissues, in agreement with our previous results (Zatelli et al. 2010a, Gagliano et al. 2013). On this basis, our data suggest that the lack of response to Everolimus in vitro may be due to the low expression levels of $\mathrm{PI} 3 \mathrm{~K} / \mathrm{AKT} / \mathrm{mTOR}$ pathway components. Our results are in contrast with a recent study showing that high p-p70S6K protein levels are associated with a worse outcome under Everolimus treatment in NET (Benslama et al. 2016). However, the latter study was preformed including a very heterogeneous group of NET of different origin. Therefore, the results obtained by Benslama and coworkers may not reflect P-NET behavior (Benslama et al. 2016).

Besides putative molecular markers, we also investigated whether P-NET clinical characteristics may differ between responders and non-responders. We found a higher ki67 index in human P-NET-R compared with P-NET-NR and a trend for a linear correlation between tumor grade and response to Everolimus, suggesting that Everolimus responsiveness may associate with a greater clinical aggressiveness of P-NET.

Even though AlphaScreen represents a very sensitive method, validated by Western blot in bronchial carcinoids (Gagliano et al. 2013), it is not widely available. Therefore, we assessed IHC for $\mathrm{p}$-AKT protein to validate the identified putative markers of response to Everolimus by means of a method that may be used also by other centers. The low number of available samples limited the significance of our findings as the correlation between p-AKT positivity by IHC and responsiveness to Everolimus in vitro did not reach statistical significance. The lack of correlation between AlphaScreen results obtained in fresh frozen tissues and IHC data for p-AKT expression is of importance. Although the fresh tissues were frozen after being stored several hours in RNAlater, which is a stabilizing RNA but does not influence protein phosphorylation, the paraffin-embedded tissues were processed routinely in the pathology lab with a variation in fixation times. The IHC protocols were performed on tissues that were fixed as large blocks and formalin is known to penetrate at $1 \mathrm{~mm} / \mathrm{h}$. During this time, phosphorylation levels of AKT may profoundly change (Baker et al. 2005), possibly explaining the different results obtained using these two different approaches. However, it is interesting to notice that p-AKT positivity by IHC was found in a P-NET-R patient who showed tumor stabilization under Everolimus treatment in vivo, suggesting that even P-NET-G3 may take advantage of this drug. On the contrary, IHC failed to demonstrate a strong p-AKT staining in a P-NET-NR who showed disease progression under Everolimus treatment in vivo. These findings suggest the presence of a good correlation between the responsiveness to Everolimus in vitro and that in vivo and provide the basis for further studies aimed at validating the identified putative markers of Everolimus responsiveness.

In conclusion, our results show that P-NET primary cultures may represent a suitable model for testing medical treatment efficacy. In addition, our data indicate that IGF1 pathway is involved in the development of resistance to Everolimus and that that IHC characterization of p-AKT may help in identifying human P-NET who can benefit from Everolimus treatment. However, these preliminary data need to be confirmed and encourage conducting a prospective multicenter study involving different groups of P-NET patients treated with Everolimus.

Published by Bioscientifica Ltd 


\section{Declaration of interest}

M C Z has received consultant fees from Novartis and Genzyme. E d U has received consultant fees from Novartis and grant support from Sanofi. The other authors have nothing to disclose concerning a possible conflict of interest.

\section{Funding}

This work was supported by grants from the Italian Ministry of Education, Research and University (FIRB RBAP11884M, RBAP1153LS), Fondazione Dott. Carlo Fornasini and Associazione Italiana per la Ricerca sul Cancro (AIRC) in collaboration with Laboratorio in rete del Tecnopolo 'Tecnologie delle terapie avanzate' (LTTA) of the University of Ferrara. The funding sources had no involvement in study design, in the collection, analysis and interpretation of data, in the writing of the report and in the decision to submit the article for publication.

\section{Author contribution statement}

$S$ Falletta wrote the manuscript, performed primary cultures and AlphaScreen experiments and helped in setting up the immunohistochemistry experiments; $S$ Partelli helped in providing the surgical specimens; C Rubini provided the paraffin-embedded tissues; D Nann performed the immunohistochemistry experiments; A Doria helped in providing the paraffin-embedded tissues; I Marinoni provided support for the immunohistochemistry experiments and reviewed the manuscript; V Polenta helped in providing the clinical data; C Di Pasquale helped in performing the primary culture experiments; E degli Uberti provided support for the clinical correlations; A Perren supervised the immunohistochemistry experiments and reviewed the manuscript; M Falconi supervised the surgical specimen provision and reviewed the manuscript and M C Zatelli wrote the manuscript, supervised the in vitro experiments and the project development.

\section{Acknowledgements}

The authors thank Dr Miguel Sampedro Nuñez for his help in the statistical evaluation.

\section{References}

Al-Kurd A, Chapchay K, Grozinsky-Glasberg S \& Mazeh H 2014 Laparoscopic resection of pancreatic neuroendocrine tumors. World Journal of Gastroenterology 20 4908-4916. (doi:10.3748/wjg. v20.i17.4908)

Baker AF, Dragovich T, Ihle NT, Williams R, Fenoglio-Preiser C \& Powis G 2005 Stability of phosphoprotein as a biological marker of tumor signaling. Clinical Cancer Research 11 4338-4340. (doi:10.1158/1078-0432.CCR-05-0422)

Benslama N, Bollard J, Vercherat C, Massoma P, Roche C, Hervieu V, Peron J, Lombard-Bohas C, Scoazec JY \& Walter T 2016 Prediction of response to everolimus in neuroendocrine tumors: evaluation of clinical, biological and histological factors. Investigational New Drugs 34 654-662. (doi:10.1007/s10637-016-0363-6)

Berardi R, Morgese F, Torniai M, Savini A, Partelli S, Rinaldi S, Caramanti M, Ferrini C, Falconi M \& Cascinu S 2016 Medical treatment for gastro-entero-pancreatic neuroendocrine tumours. World Journal of Gastrointestinal Oncology 8 389-401. (doi:10.4240/ wjgs.v8.i5.389)

Capdevila J, Salazar R, Halperín I, Abad A \& Yao JC 2011 Innovations therapy: mammalian target of rapamycin (mTOR) inhibitors for the treatment of neuroendocrine tumors. Cancer and Metastatis Review 30 27-34. (doi:10.1007/s10555-011-9290-3)

Fazio N 2015 Neuroendocrine tumors resistant to mammalian target of rapamycin inhibitors: A difficult conversion from biology to the clinic. World Journal of Clinical Oncology 6 194-197. (doi:10.5306/ wjco.v6.i6.194)

Gagliano T, Bellio M, Gentilin E, Molè D, Tagliati F, Schiavon M, Cavallesco NG, Andriolo LG, Ambrosio MR, Rea F, et al. 2013 mTOR, p70S6K, AKT, and ERK1/2 levels predict sensitivity to mTOR and $\mathrm{PI} 3 \mathrm{~K} / \mathrm{mTOR}$ inhibitors in human bronchial carcinoids. EndocrineRelated Cancer 20 463-475. (doi:10.1530/ERC-13-0042)

Gentilin E, Di Pasquale C, Rossi M, Tagliati F, Gagliano T, Rossi R, Pelizzo M, Merante Boschin I, degli Uberti EC \& Zatelli MC 2015 Igf-I influences everolimus activity in medullary thyroid carcinoma. Frontiers in Endocrinology 6 63. (doi:10.3389/fendo.2015.00063)

Halfdanarson TR, Rabe KG, Rubin J \& Petersen GM 2008 Pancreatic neuroendocrine tumors (PNETs): incidence, prognosis and recent trend toward improved survival. Annals of Oncology 19 1727-1733. (doi:10.1093/annonc/mdn351)

Höpfner M, Schuppan D \& Scherübl H 2008 Treatment of gastrointestinal neuroendocrine tumors with inhibitors of growth factor receptors and their signaling pathways: recent advances and future perspectives. World Journal of Gastroenterology 14 2461-2473. (doi:10.3748/wjg.14.2461)

Jiang BH \& Liu LZ 2008 Role of mTOR in anticancer drug resistance: perspectives for improved drug treatment. Drug Resistance Updates 11 63-76. (doi:10.1016/j.drup.2008.03.001)

Missiaglia E, Dalai I, Barbi S, Beghelli S, Falconi M, della Peruta M, Piemonti L, Capurso G, Di Florio A, delle Fave G, et al. 2010 Pancreatic endocrine tumors: expression profiling evidences a role for AKT-mTOR pathway. Journal of Clinical Oncology 28 245-255. (doi:10.1200/JCO.2008.21.5988)

Molè D, Gagliano T, Gentilin E, Tagliati F, Pasquali C, Ambrosio MR, Pansini G, degli Uberti EC \& Zatelli MC 2011 Targeting protein kinase $\mathrm{C}$ by Enzastaurin restrains proliferation and secretion in human pancreatic endocrine tumors. Endocrine-Related Cancer 18 439-450. (doi:10.1530/ERC-11-0055)

O'Reilly KE, Rojo F, She QB, Solit D, Mills GB, Smith D, Lane H, Hofmann F, Hicklin DJ, Ludwig DL, et al. 2006 mTOR inhibition induces upstream receptor tyrosine kinase signaling and activates Akt. Cancer Research 66 1500-1508. (doi:10.1158/0008-5472.CAN05-2925)

Pavel M, O'Toole D, Costa F, Capdevila J, Gross D, Kianmanesh R, Krenning E, Knigge U, Salazar R, Pape UF, et al. 2016 ENETS consensus guidelines update for the management of distant metastatic disease of intestinal, pancreatic, bronchial neuroendocrine neoplasms (NEN) and NEN of unknown primary site. Neuroendocrinology 103 172-185. (doi:10.1159/000443167)

Qian ZR, Ter-Minassian M, Chan JA, Imamura Y, Hooshmand SM, Kuchiba A, Morikawa T, Brais LK, Daskalova A, Heafield R, et al. 2013 Prognostic significance of MTOR pathway component expression in neuroendocrine tumors. Journal of Clinical Oncology $\mathbf{3 1}$ 3418-3425. (doi:10.1200/JCO.2012.46.6946)

Righi L, Volante M, Rapa I, Tavaglione V, Inzani F, Pelosi G \& Papotti M 2010 Mammalian target of rapamycin signaling activation patterns in neuroendocrine tumors of the lung. Endocrine-Related Cancer $\mathbf{1 7}$ 977-987. (doi:10.1677/ERC-10-0157)

Ro C, Chai W, Yu VE \& Yu R 2013 Pancreatic neuroendocrine tumors: biology, diagnosis, and treatment. Chinese Journal of Cancer 32 312-324. (doi:10.5732/cjc.012.10295)

Singh S, Dey C, Kennecke H, Kocha W, Maroun J, Metrakos P, Mukhtar T, Pasieka J, Rayson D, Rowsell C, et al. 2015 Consensus recommendations for the diagnosis and management of pancreatic neuroendocrine tumors: guidelines from a Canadian National Expert Group. Annals of Surgical Oncology 22 2685-2699. (doi:10.1245/ s10434-014-4145-0) http://erc.endocrinology-journals.org DOI: 10.1530/ERC-16-0329
(C) 2016 Society for Endocrinology Printed in Great Britain 
Tagliati F, Zatelli MC, Bottoni A, Piccin D, Luchin A, Culler MD \& degli Uberti EC 2006 Role of complex cyclin D1/Cdk4 in somatostatin subtype 2 receptor-mediated inhibition of cell proliferation of a medullary thyroid carcinoma cell line in vitro. Endocrinology 147 3530-3538. (doi:10.1210/en.2005-1479)

Vallea J, Eatock M, Clueit B, Zahava G, Roxanne F \& Mitchell S 2014 A systematic review of non-surgical treatments for pancreatic neuroendocrine tumours. Cancer Treatment Reviews 40 376-389. (doi:10.1016/j.ctrv.2013.08.007)

von Wichert G, Jehle PM, Hoeflich A, Koschnick S, Dralle H, Wolf E, Wiedenmann B, Boehm BO, Adler G \& Seufferlein T 2000 Insulinlike growth factor-I is an autocrine regulator of chromogranin A secretion and growth in human neuroendocrine tumor cells. Cancer Research $604573-4581$.

Wolin EM 2014 Long-term Everolimus treatment of patients with pancreatic neuroendocrine tumors. Chemotherapy 60 143-150. (doi:10.1159/000369780)

Yao JC, Shah MH, Ito T, Bohas CL, Wolin EM, Van Cutsem E, Hobday TJ, Okusaka T, Capdevila J, de Vries EG, et al. 2011 Everolimus for advanced pancreatic neuroendocrine tumors. New England Journal of Medicine 364 514-523. (doi:10.1056/ NEJMoa1009290)
Zatelli MC, Piccin D, Tagliati F, Bottoni A, Luchin A, Vignali C, Margutti A, Bondanelli M, Pansini GC, Pelizzo MR, et al. 2006 Selective activation of somatostatin receptor subtypes differentially modulates secretion and viability in human medullary thyroid carcinoma primary cultures: potential clinical perspectives. Journal of Clinical Endocrinology and Metabolism 91 2218-2224. (doi:10.1210/ jc.2006-0334)

Zatelli MC, Minoia M, Martini C, Tagliati F, Ambrosio MR, Schiavon M, Buratto M, Calabrese F, Gentilin E, Cavallesco G, et al. 2010 a Everolimus as a new potential antiproliferative agent in aggressive human bronchial carcinoids. Endocrine-Related Cancer 17 719-729. (doi:10.1677/ERC-10-0097)

Zatelli MC, Gentilin E, Daffara F, Tagliati F, Reimondo G, Carandina G, Ambrosio MR, Terzolo M \& degli Uberti EC $2010 b$ Therapeutic concentrations of mitotane (o,p'-DDD) inhibit thyrotroph cell viability and TSH expression and secretion in a mouse cell line model. Endocrinology 151 2453-2461. (doi:10.1210/ en.2009-1404)

Zatelli MC, Fanciulli G, Malandrino P, Ramundo V, Faggiano A, Colao A $\&$ NIKE Group 2016 Predictive factors of response to mTOR inhibitors in neuroendocrine tumours. Endocrine-Related Cancer 23 R173-R183. (doi:10.1530/ERC-15-0413)

Received in final form 21 September 2016

Accepted 3 October 2016

Accepted Preprint published online 3 October 2016 